\title{
A versatile two-part system for on-line measurement of multiple response parameters
}

\author{
DAVID MYERS and MICHAEL M. PATTERSON \\ Kirksville College of Osteopathic Medicine, Kirksville, Missouri 63501
}

\begin{abstract}
A two-part system for digital measurement of such diverse response parameters as latency, pulse width, amplitude, and events per unit time is described. The mainframe consists of counters with light-emitting diode (LED) numeric readouts and a quartz crystal controlled internal time base. It also contains all power supply requirements for both the mainframe electronics and the second part of the system, the plug-ins. Each plug-in is designed to use the main unit counters, timers, readouts, and power supplies to measure the desired parameter. Thus, each numeric readout channel of the mainframe may be used to measure various parameters simply by changing the plug-in attached to it. The unit has proved to be very useful in reducing experimenter error in measuring response parameters in classical nictitating membrane conditioning.
\end{abstract}

Many studies require not only response occurrence or nonoccurrence to be recorded, but also onset latency and peak response characteristics. Other response variables, such as events per unit time, are also often recorded. Under these circumstances, the volume of data often does not justify computer analysis, while hand scoring of the response characteristics is not only laborious but often not particularly accurate. Reliability of measurements between scorers is often a problem due to to unnoticed differences in criteria of response parameters.

We have developed a transistorized system for measuring various response characteristics that can then be recorded from digital readouts. This system is ideal for laboratories needing an inexpensive device for response measures and can be interfaced with other systems by appropriate modifications. The system described here was designed to measure response characteristics during classical conditioning of the cat and rabbit nictitating membrane as well as multiple unit activity from the animal's brain. It has been in use for over a year with no serious problems.

\section{SYSTEM DESCRIPTION}

Basically, the measurement system consists of a mainframe and plug-ins as shown schematically in Figure 1. The mainframe contains four circuit functions: (1) power supplies, (2) clock circuits, (3) counter circuits, and (4) readout circuits. The power supply provides regulated and overload protected voltages

The development of this equipment was made possible by grants from the American Osteopathic Association Bureau of Research and by NINCDS Grant NS10647 to the second author. Reprint requests should be sent to Michael M. Patterson at the College of Osteopathic Medicine, Ohio University, Athens, Ohio 45701 . of $+5,+15$, and -15 , and supplies six channels of counters, readouts, and plug-ins, as well as the main clock unit. The clock consists of a $100-\mathrm{kHz}$ quartz crystal controlled oscillator, driving decade dividers to provide clock rates of $10^{4}, 10^{3}, 10^{2}, 10^{1}$, and $10^{0} \mathrm{~Hz}$. Each mainframe channel contains a 000-999 base-10 counter with LED readouts and drivers. The accuracy of the system is at least $1 \%$ and the digital LED readouts provide easy experimenter access to the data.

The plug-ins form the interface between the main unit and the responses to be measured. Each plug-in has all the power supply voltages, counter inputs, and clock outputs at its disposal through its plug-in receptacle on the mainframe. Each type of plug-in is compatible with any channel of the mainframe without modification and varies in complexity with the difficulty of converting input signals to pulses useful for the mainframe counters.

Described below are the theory of operation and circuit function of the mainframe components as well as three plug-ins used in our laboratory, specifically for counting, timing, and amplitude measurement. The plug-ins described are designed to be compatible with Grass polygraph outputs and Coulbourn sequencing equipment but can be modified to correspond to other equipment by changing voltage divider ratios.

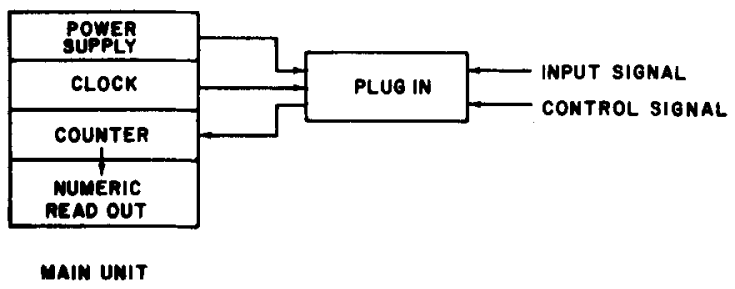

Figure 1. Basic flow diagram of the mainframe and plug-in systems used in the equipment. 


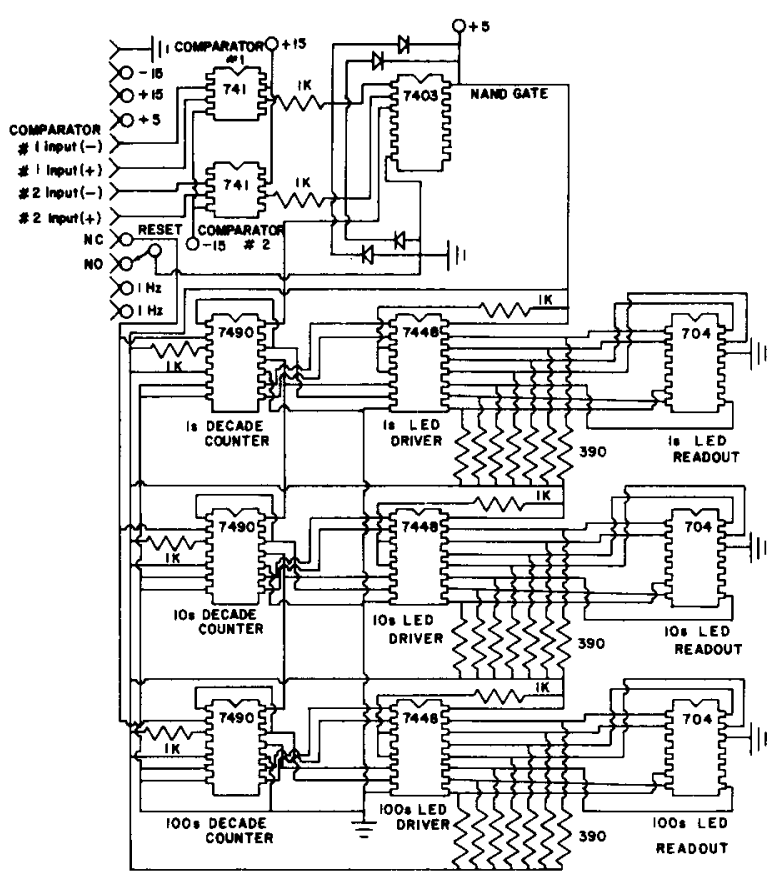

Figure 2. Schematic diagram of the mainframe counter unit.

\section{Mainframe}

Counters. All input parameters are converted in the plug-ins to pulses that can be counted or timed by the mainframe counters. Each channel is thus a digital counter driving corresponding LED readouts. Each counter is driven by a NAND gate with a voltage comparator connected to each of the two gate inputs. If the voltage at the positive input of a comparator is more positive than the voltage at the negative input, then a positive level is presented to the corresponding gate input. If and only if both gate inputs have positive levels, a count is performed. Thus, for a count to be performed, each comparator must have a more positive gate voltage on its positive input than on its negative input. In order for another count to be performed, the input voltages must first change to a no-count configuration and then back to a count state.

The counter is composed of 7400 series digital integrated circuits, driving LED numeric readouts as shown in Figure 2. The counting is performed by three cascaded 7490 decade counters. Each decade counter drives a 7448 LED driver, which in turn drives a 704 LED. The 7490 in the one position is driven by the output of one section of a 7403 quad nand gate. The inputs of the nand gate are connected to the outputs of 741 operational amplifiers (op amps) used as comparators, as mentioned above. The op amp output levels are clipped to provide logic level compatibility with the 7403 inputs. The only access to a mainframe counter input is through the comparator inputs. This configuration provides extreme flexibility in the magnitude and dc level of the pulses to be counted. The peak-to-peak amplitude can be as small as $10 \mathrm{mV}$ or as large as $30 \mathrm{~V}$. The dc levels can range from -15 to $+15 \mathrm{~V}$. In no case, however, should the sum of the peak-to-peak level plus the dc level ever exceed -15 or $+15 \mathrm{~V}$ with respect to ground, or the comparator inputs will be damaged. The 741 inputs also provide input impedance on the order of $300,000 \mathrm{ohms}$.

The counter is reset by grounding the 7490 decade counter reset input. This is accomplished by switch or relay contacts depending on the builder's preference. Each channel should be reset by separate contacts.

Clock. The quartz crystal controlled clock (Figure 3) provides pulses at fixed intervals that can be applied to one of the comparator inputs through the internal wiring of a plug-in.

The quartz crystal oscillator is a Hartley $100-\mathrm{kHz}$ oscillator using a FET(Q9). This output is capacitor coupled to a 7413 Schmitt trigger, which in turn drives a series of cascaded 7490 decade counters. This provides outputs of $100 \mathrm{kHz}$ to $1 \mathrm{~Hz}$ in 1 -decade increments. In this system, only the $1-\mathrm{Hz}$ and $1 \mathrm{kHz}$ signals are provided to the plug-in receptacles because seconds and milliseconds are the units of time measurement needed in the current work.

Power supply. The power supply (Figure 4) is composed of three supplies providing different voltages from a common ground. This power supply is capable of providing power for six counters, the clock, and associated plug-ins. The +15 - and $-15-\mathrm{V}$ supplies are overload protected with current limited to $500 \mathrm{~mA}$. The regulation is accomplished with a Darlington series regulator connected to a $16-\mathrm{V}$ Zener reference. The $5-\mathrm{V}$ supply is regulated by a SI $3554 \mathrm{M}$ hybrid regulator, which is current limited at $3 \mathrm{~A}$. The total power consumption at maximum rated output is less than $70 \mathrm{~W}$.

\section{Plug-Ins}

Events per unit time, or pulse duration. In this

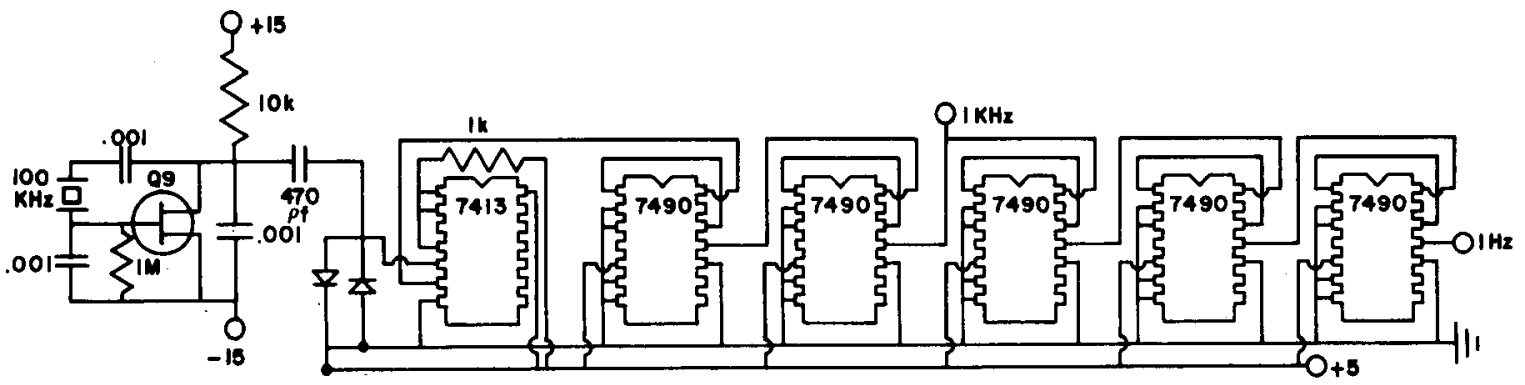

Figure 3. Sehematic diagram of the internal clock. 

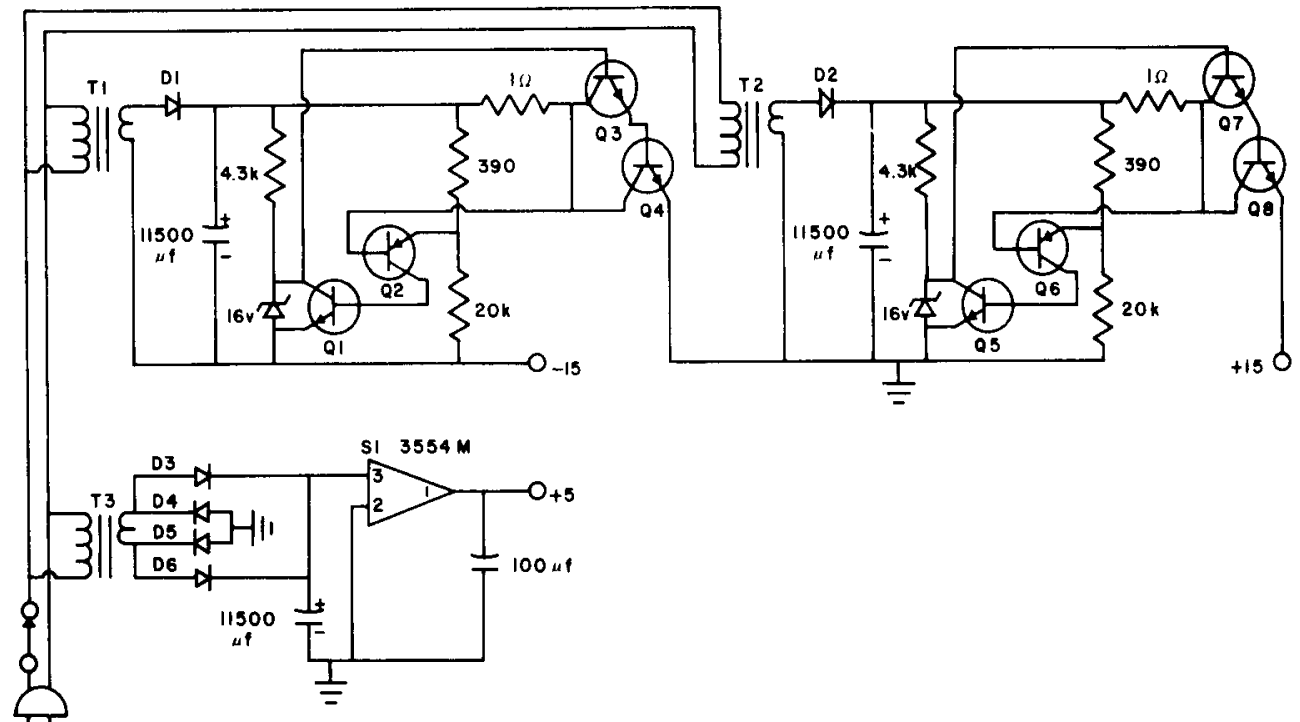

Figure 4. Schematic diagram of the power supplies.

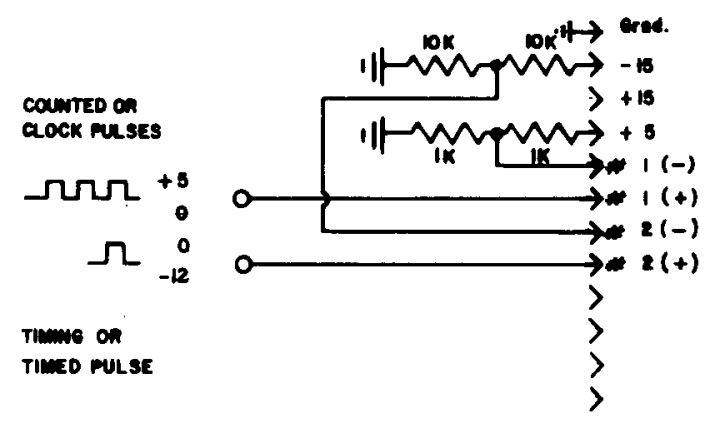

Figure 5. Schematic of the events per unit time plug-in.

plug-in, the inputs consist of one positive timing pulse of fixed duration and an unknown number of pulses from the output of a brainwave spike analyzer to be counted during the timing pulse. The plug-in (Figure 5) consists of two voltage dividers. One provides a voltage between the minimum and maximum values of a spike analyzer's output pulses (counted or clock pulses). The other voltage divider provides a voltage between the minimum and maximum levels of the fixed-length (timing) pulse. Counted and timing pulses are applied to the positive comparator inputs of the mainframe, while the voltage divider levels are applied to the corresponding negative inputs. When both pulses are positive, a count is made and counting is continued with each fluctuation of the data pulse until the timing pulse terminates.

Alternatively, by providing fixed-interval clock pulses to the counting input, variable-length pulses can be timed. To accomplish this, the voltage divider level of Comparator E1's negative input is set between the maximum and minimum levels of the main unit clock pulse (for example, the millisecond clock input) that is applied to the positive input. Comparator 2's negative input voltage divider is set between the peak levels of the pulse to be timed, and the pulse is applied to the positive input of Comparator 2 . Thus, the plug-in can function either as an events per unit time counter or as a pulse length timer.

Peak amplitude. This plug-in measures the peak amplitude of response waveforms taken from the driver output of Grass polygraph amplifiers. Two inputs are

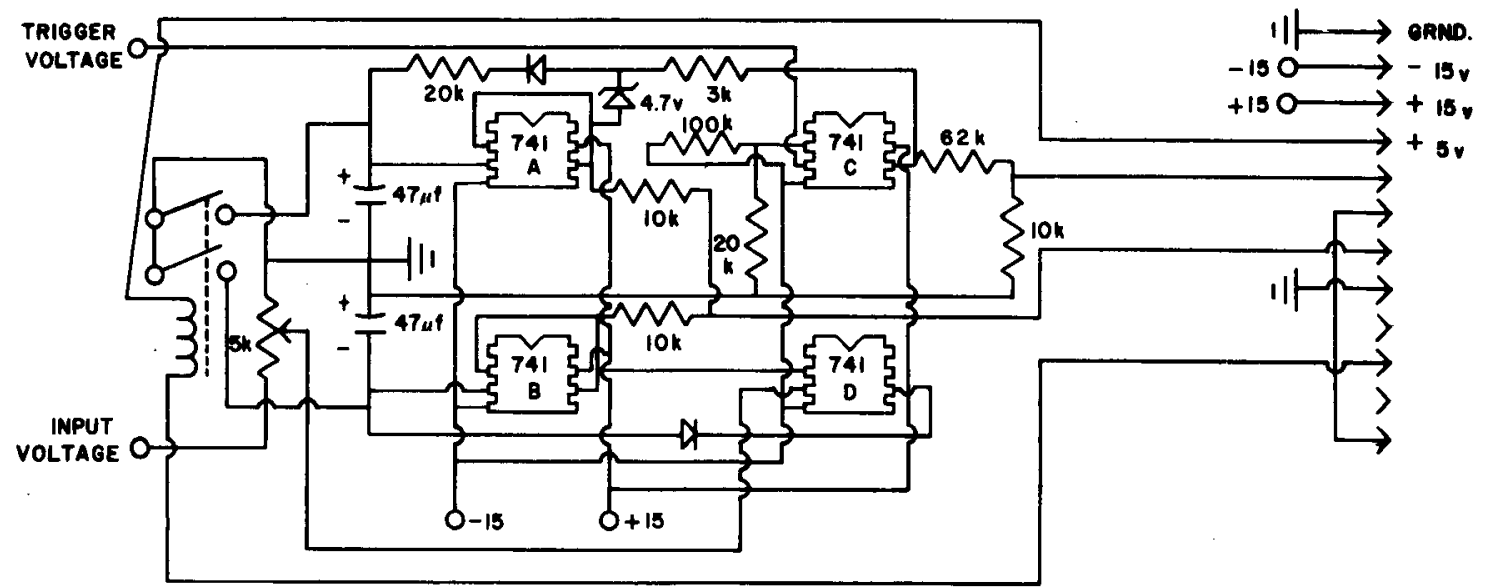

Figure 6. Schematic of the peak amplitude detector plug-in. 


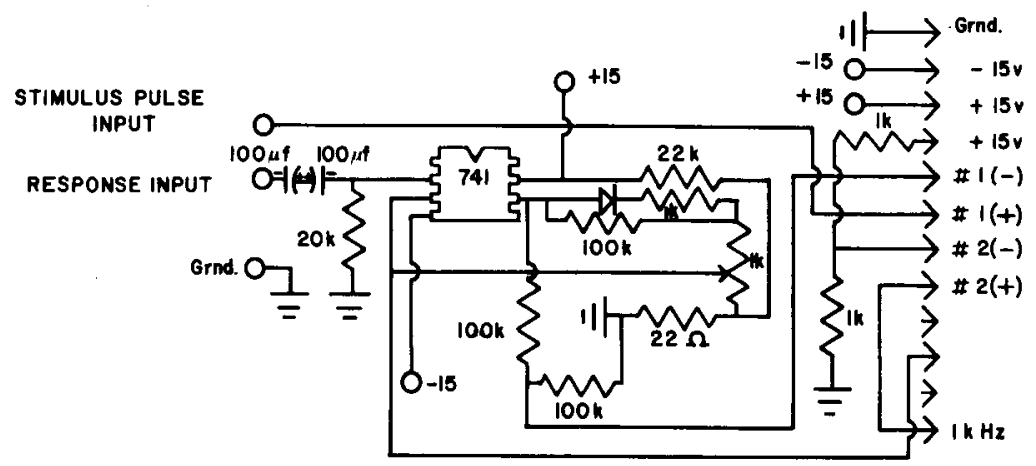

Figure 7. Schematic of the latency to response onset plug-in.

provided, as seen in Figure 6. One is the response waveform, which varies in amplitude, and the other is a positive pulse, which starts when the trial is over. It is necessary that the plug-in remember the peak amplitude voltage to convert it to a pulse width proportional to that voltage. The first objective is accomplished with a sample-and-hold peak detector circuit and the second by comparing the peak detector output with a linearly increasing ramp voltage. The ramp voltage and the counter both begin with the trial end pulse. The counter counts 1 -msec timing pulses until the absolute ramp voltage is greater than the absolute sample-and-hold voltage, at which point the counter stops.

Op amp 741A (Figure 6) generates the linear ramp voltage by acting as a current source to the negative

Table 1

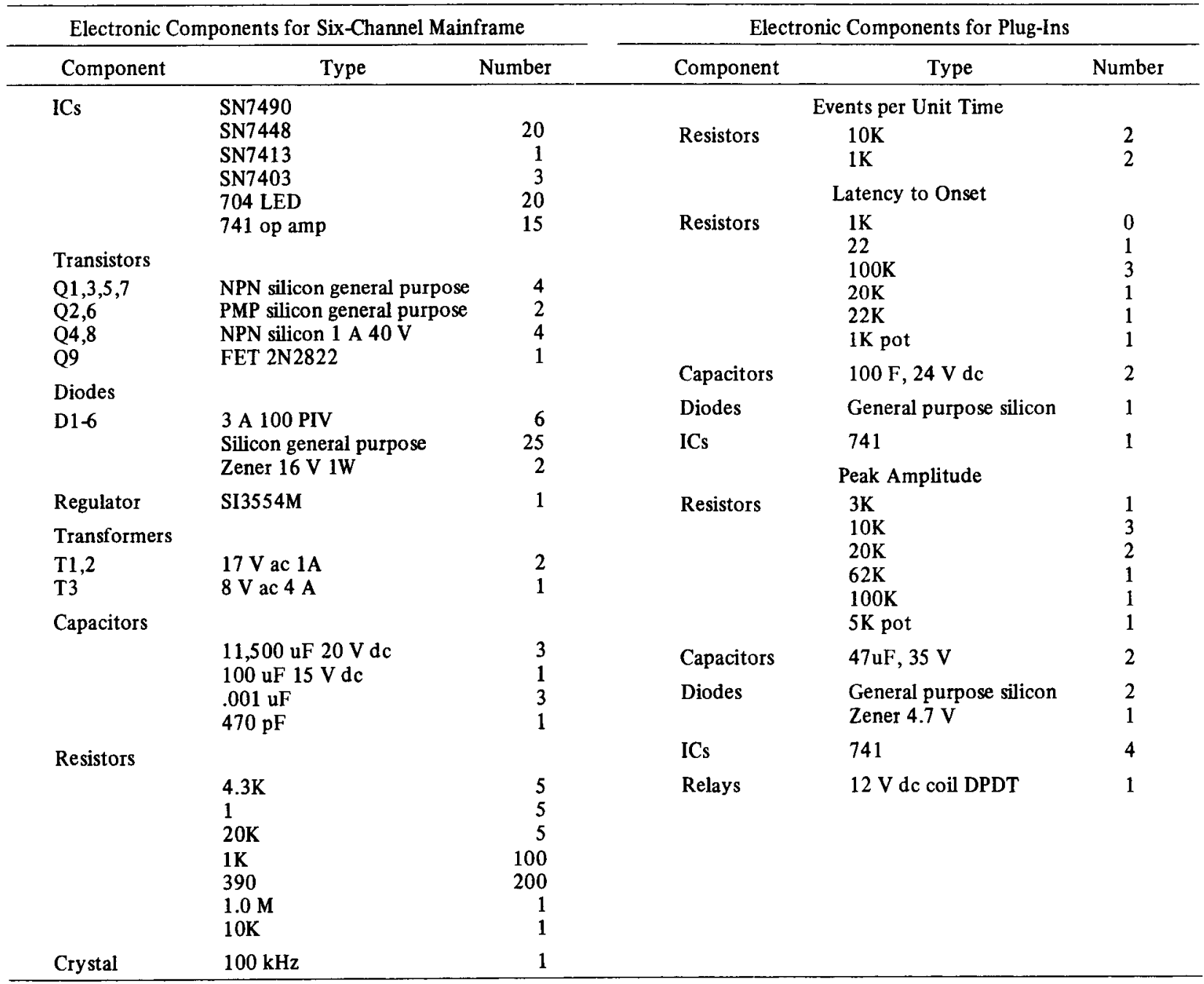


ground capacitor. Op amp $741 \mathrm{C}$ turns on the ramp generator when the positive trial end pulse is present. Op amps 741B and D sample charge and prevent discharge of the positive ground voltage memory capacitor, thus forming a sample-and-hold peak detector. The double pole relay discharges the memory and ramp capacitors. It must be closed for approximately $5 \mathrm{sec}$ just prior to the trial, but not during it, to assure complete discharge of the memory and ramp capacitors. A response amplitude of $-2.8 \mathrm{~V}$ with no attenuation results in a reading of 999 . By adjusting the 5-Kohm attenuator potentiometer, one can achieve any reading from 000 to 999 with a $-2.8-\mathrm{V}$ amplitude signal. If a reading calibrated to millimeters of deflection is desired, the potentiometer is simply adjusted until the reading corresponds to a standard response height measured in millimeters. If the input voltage is not sufficient to produce the desired count, alteration of the feedback network on 741B can be used to increase the input gain of the sample-and-hold circuit.

Latency to onset. This plug-in (Figure 7) measures elapsed time from stimulus onset to response onset. One input consists of a positive pulse that is initiated by stimulus onset and is longer than the maximum expected latency. The other input is a negative going waveform corresponding to the response, which may oscillate above and below the baseline after initial response. The positive stimulus waveform initiates counting of timing pulses, and the initial negative response waveform triggers a positive output from the op amp, which stops counting of timing pulses. The gain of the op amp is very high when the output is negative, thus allowing very small responses to stop the counting of timing pulses. The gain is essentially unity with a positive output, thus preventing the unit from resuming the count when the response returns to baseline or below. This gain differential is accomplished by shunting the $100 \mathrm{~K}$ feedback resistor with a diode that conoucts only when the output is positive. Capacitance coupling of response input allows slow baseline changes without manual readjustment. The $1 \mathrm{~K}$ potentiometer adjusts the response waveform amplitude necessary to stop the timing pulse count.

\section{CONSTRUCTION}

Table 1 gives the components needed for construction of the equipment described. The electronic components for a six-channel base unit total approximately $\$ 125$. Depending on materials used and mounting configuration, hardware such as switches, relays, heat sinks, chassis, wire, sockets, and so on, will total about $\$ 75$. Thus, the materials cost is approximately $\$ 200$ for a six-channel mainframe, while plug-in cost can vary from $\$ 2$ to $\$ 25$ per unit. The following points should be considered in construction of the equipment: (1) All switches may be replaced by relays if desired. (2) System reset should be accomplished just prior to trial commencement to avoid spurious triggering. (3) By-pass capacitors should be attached at intervals along power supply lines to ground. (4) Voltage divider switching levels may be changed to suit one's own equipment. (5) Input lines should be shielded and as short as practical. (6) IC sockets are recommended. (7) Mainframe plug-in receptacles should be spaced far enough apart to allow room for plug-ins.

(Received for publication November 18, 1977; accepted December 16, 1977.) 